FACTA UNIVERSITATIS (NIŠ)

Ser. Math. Inform. Vol. 34, No 3 (2019), 553-561

https://doi.org/10.22190/FUMI1903553U

\title{
ON BIVARIATE RETARDED INTEGRAL INEQUALITIES AND THEIR APPLICATIONS
}

\author{
Fuat Usta and Mehmet Zeki Sarıkaya
}

(C) 2019 by University of Niš, Serbia | Creative Commons Licence: CC BY-NC-ND

\begin{abstract}
In this paper, we obtain some retarded integral inequalities in two independent variables which can be used as tools in the theory of partial differential and integral equations with time delays. The presented inequalities are of new forms compared with the existing ones so far in the literature. In order to illustrate the validity of the theorems we give one application for them for the solution to certain fractional order differential equations.
\end{abstract}

Keywords: integral inequalities; differential equations; time delay.

\section{Introduction}

As it is well known integral inequalities play a significant role in the qualitative analysis of differential and integral equations theory. Over the years, various investigators have discovered many useful integral inequalities in order to achieve a diversity of desired goals, see [1]-[12] and the references given therein. In a recent paper [8] Pachpatte presented a retarded inequality which has very good characters. A large number of papers have been presented dealing with various extensions and generalizations of this inequality. Some of the results may be found in [8], but let us first recall the main results of $[8]$ as follows:

In what follows, $\mathbb{R}$ denotes a set of real numbers, $\mathbb{R}_{+}=[0, \infty), J_{1}=\left[x_{0}, X\right), J_{2}=$ $\left[y_{0}, Y\right)$ are given subsets of $\mathbb{R}, \Delta=J_{1} \times J_{2}$ and ' denotes the derivative.

Theorem 1.1. Let $u(x, y), a(x, y) \in C\left(\Delta, \mathbb{R}_{+}\right), \quad b(x, y, s, t) \in C\left(\Delta^{2}, \mathbb{R}_{+}\right)$, for $x_{0} \leq s \leq x \leq X, y_{0} \leq t \leq y \leq Y, \alpha(x) \in C^{1}\left(J_{1}, J_{1}\right), \beta(y) \in C^{1}\left(J_{2}, J_{2}\right)$ be non-decreasing with $\alpha(x) \leq x$ on $J_{1}, \beta(y) \leq y$ on $J_{2}$ and $k \geq 0$ be a constant.

Received September 21, 2018; accepted October 02, 2018

2010 Mathematics Subject Classification. Primary 26D15; Secondary 26A51, 26A33, 26A42 
( $\left.A_{1}\right)$ If

$u(x, y) \leq k+\int_{\alpha\left(x_{0}\right)}^{\alpha(x)} \int_{\beta\left(y_{0}\right)}^{\beta(y)}\left[a(s, t) u(s, t)+\int_{\alpha\left(x_{0}\right)}^{s} \int_{\beta\left(y_{0}\right)}^{t} b(s, t, \sigma, \eta) u(\sigma, \eta) d \sigma d \eta\right] d t d s$,

for $(x, y) \in \Delta$, then

$$
u(x, y) \leq k \exp (A(x, y))
$$

for $(x, y) \in \Delta$, where

$$
A(x, y)=\int_{\alpha\left(x_{0}\right)}^{\alpha(x)} \int_{\beta\left(y_{0}\right)}^{\beta(y)}\left[a(s, t)+\int_{\alpha\left(x_{0}\right)}^{s} \int_{\beta\left(y_{0}\right)}^{t} b(s, t, \sigma, \eta) d \sigma d \eta\right] d t d s,
$$

for $(x, y) \in \Delta$.

$\left(A_{2}\right)$ Let $g \in C\left(\mathbb{R}_{+}, \mathbb{R}_{+}\right)$be a non-decreasing function with $g(u)>0$ for $u>0$. If

$u(x, y) \leq k+\int_{\alpha\left(x_{0}\right)}^{\alpha(x)} \int_{\beta\left(y_{0}\right)}^{\beta(y)}\left[a(s, t) g(u(s, t))+\int_{\alpha\left(x_{0}\right)}^{s} \int_{\beta\left(y_{0}\right)}^{t} b(s, t, \sigma, \eta) g(u(\sigma, \eta)) d \sigma d \eta\right] d t d s$,

for $(x, y) \in \Delta$, then for $x_{0} \leq x \leq x_{1}, y_{0} \leq y \leq y_{1}$,

$$
u(x, y) \leq G^{-1}[G(k)+A(x, y)]
$$

where $A(x, y)$ is defined by (1.3), $G^{-1}$ is the inverse function of

$$
G(r)=\int_{r_{0}}^{r} \frac{d s}{g(s)}, r>0, r_{0}>0
$$

and $x_{1} \in J_{1}, y_{1} \in J_{2}$ are chosen so that

$$
G(k)+A(x, y) \in \operatorname{Dom}\left(G^{-1}\right),
$$

for all $x$ and $y$ lying in $\left[x_{0}, x\right]$ and $\left[y_{0}, y\right]$ respectively.

The purpose of this paper is to explore two independent retarded versions of the above integral inequalities which can be used as tools in the theory of partial differential and integral equations with time delays. Applications are also given to convey the significance of our results. 


\section{Main Results}

The first section of this paper will present some new non-linear retarded integral inequalities in two independent variables which can be used as effective tools in the study on non-linear partial differential equations with time delay.

Theorem 2.1. If $u(x, y), p(x, y), a(x, y)$ are real valued non-negative continuous functions and $u(x, y) \geq 2 p(x, y)$ is defined for $x \geq 0, y \geq 0, b(x, y, s, t)$ are continuous non-decreasing in $x$ and $y$ for $t, s .0 \leq \alpha(x) \leq x, 0 \leq \beta(y) \leq y, \alpha^{\prime}(x), \beta^{\prime}(y) \geq 0$ are real valued continuous functions defined for $x \geq 0, y \geq 0$, that satisfy

$$
u(x, y) \leq p(x, y)+\int_{0}^{\alpha(x)} \int_{0}^{\beta(y)}\left[a(s, t) u(s, t)+\int_{0}^{s} \int_{0}^{t} b(s, t, \sigma, \eta) u(\sigma, \eta) d \sigma d \eta\right] d t d s
$$

then

$$
u(x, y) \leq p(x, y) \times\left(1+e \int_{0}^{\alpha(x)} \int_{0}^{\beta(y)}\left[a(s, t)+\int_{0}^{s} \int_{0}^{t} b(s, t, \sigma, \eta) d \sigma d \eta\right] d t d s\right)
$$

Proof. First of all let $z(x, y)$ denote the function on the right hand side of 2.1 , that is,

$$
z(x, y)=\int_{0}^{\alpha(x)} \int_{0}^{\beta(y)}\left[a(s, t) u(s, t)+\int_{0}^{s} \int_{0}^{t} b(s, t, \sigma, \eta) u(\sigma, \eta) d \sigma d \eta\right] d t d s
$$

then $z(0, y)=z(x, 0)=0$ and our assumption on $a, b, u, \alpha$ and $\beta$ imply that $z$ is a non-decreasing positive function for $x \geq 0, y \geq 0$ and $x \in\left[0, T_{1}\right], y \in\left[0, T_{2}\right]$ we have

$$
\begin{aligned}
z_{x y}(x, y) & =\alpha^{\prime}(x) \beta^{\prime}(y)\left[a(\alpha(x), \beta(y)) u(\alpha(x), \beta(y))+\int_{0}^{\alpha(x)} \int_{0}^{\beta(y)} b(\alpha(x), \beta(y), \sigma, \eta) u(\sigma, \eta) d \sigma d \eta\right] \\
\leq & \alpha^{\prime}(x) \beta^{\prime}(y)[a(\alpha(x), \beta(y))(p(\alpha(x), \beta(y))+z(\alpha(x), \beta(y))) \\
& \left.+\int_{0}^{\alpha(x)} \int_{0}^{\beta(y)} b(\alpha(x), \beta(y), \sigma, \eta)(p(\sigma, \eta)+z(\sigma, \eta)) d \sigma d \eta\right] .
\end{aligned}
$$

Then by rearranging the above inequality we obtain

$$
\begin{aligned}
& z_{x y}(x, y) \leq z\left(T_{1}, T_{2}\right)\left(\alpha^{\prime}(x) \beta^{\prime}(y)\left[a(\alpha(x), \beta(y))+\int_{0}^{\alpha(x)} \int_{0}^{\beta(y)} b(\alpha(x), \beta(y), \sigma, \eta) d \sigma d \eta\right]\right) \\
& +\left(\alpha^{\prime}(x) \beta^{\prime}(y)\left[a(\alpha(x), \beta(y)) p\left(\alpha(x), \beta(y)+\int_{0}^{\alpha(x)} \int_{0}^{\beta(y)} b(\alpha(x), \beta(y), \sigma, \eta) p(\sigma, \eta) d \sigma d \eta\right]\right) .\right.
\end{aligned}
$$


As $0 \leq \alpha(x) \leq x$ and $0 \leq \beta(y) \leq y$ and $z(x, y)$ is non-decreasing with respect to $x$, $y$ we get

$$
\frac{z_{x y}(x, y)}{z\left(T_{1}, T_{2}\right)} \leq 2\left(\frac{\partial^{2}}{\partial x \partial y} \int_{0}^{\alpha(x)} \int_{0}^{\beta(y)}\left[a(s, t)+\int_{0}^{s} \int_{0}^{t} b(s, t, \sigma, \eta) d \sigma d \eta\right] d t d s\right) .
$$

On the other hand,

$$
\frac{\partial}{\partial y}\left(\frac{z_{x}(x, y)}{z\left(T_{1}, T_{2}\right)}\right) \leq \frac{z_{x y}(x, y)}{z\left(T_{1}, T_{2}\right)}
$$

From (2.3) and (2.4), we have

$$
\frac{\partial}{\partial y}\left(\frac{z_{x}(x, y)}{z\left(T_{1}, T_{2}\right)}\right) \leq 2\left(\frac{\partial^{2}}{\partial x \partial y} \int_{0}^{\alpha(x)} \int_{0}^{\beta(y)}\left[a(s, t)+\int_{0}^{s} \int_{0}^{t} b(s, t, \sigma, \eta) d \sigma d \eta\right] d t d s\right)
$$

Integrating both sides of the above inequality with respect to $y$ from 0 to $y$, we get

$$
\frac{z_{x}(x, y)}{z\left(T_{1}, T_{2}\right)} \leq 2\left(\frac{\partial}{\partial x} \int_{0}^{\alpha(x)} \int_{0}^{\beta(y)}\left[a(s, t)+\int_{0}^{s} \int_{0}^{t} b(s, t, \sigma, \eta) d \sigma d \eta\right] d t d s\right)
$$

then again integrating the above inequality with respect to $x$ from 0 to $x$ we obtain

$$
\ln \left|z\left(T_{1}, T_{2}\right)\right| \leq \ln \left|p\left(T_{1}, T_{2}\right)\right|+\int_{0}^{\alpha(x)} \int_{0}^{\beta(y)}\left[a(s, t)+\int_{0}^{s} \int_{0}^{t} b(s, t, \sigma, \eta) d \sigma d \eta\right] d t d s
$$

for $x \in\left[0, T_{1}\right], y \in\left[0, T_{2}\right]$. Thus we have

$$
z\left(T_{1}, T_{2}\right) \leq p\left(T_{1}, T_{2}\right) \times e^{\int_{0}^{\alpha(x)} \int_{0}^{\beta(y)}\left[a(s, t)+\int_{0}^{s} \int_{0}^{t} b(s, t, \sigma, \eta) d \sigma d \eta\right] d t d s} .
$$

Let $x=T_{1}, y=T_{2}$ in $(2.5)$, we obtain

$$
z\left(T_{1}, T_{2}\right) \leq p\left(T_{1}, T_{2}\right) \times e^{\int_{0}^{\alpha\left(T_{1}\right) \beta\left(T_{2}\right)}} \int_{0}\left[a(s, t)+\int_{0}^{s} \int_{0}^{t} b(s, t, \sigma, \eta) d \sigma d \eta\right] d t d s
$$

From the definition of $z(x, y)$, we have $u(x, y) \leq p(x, y)+z(x, y)$. As a result, we get the required inequality in (2.2).

Corollary 2.1. Assume that $a, b, \alpha, \beta$ are as in Theorem 2.1 and $p(x, y) \equiv p>0$, if $u \in C\left(\mathbb{R}_{+} \times \mathbb{R}_{+}, \mathbb{R}_{+}\right)$satisfying (2.1), then

$$
u(x, y) \leq p+p e^{\int_{0}^{\alpha(x)} \int_{0}^{\beta(y)}\left[a(s, t)+\int_{0}^{s} \int_{0}^{t} b(s, t, \sigma, \eta) d \sigma d \eta\right] d t d s}, \quad x \geq 0, y \geq 0 .
$$


Corollary 2.2. Assume that $a, b, \alpha, \beta$ are as in Theorem 2.1 and $p(x, y) \equiv p>0$. Suppose $u \in C\left(\mathbb{R}_{+} \times \mathbb{R}_{+}, \mathbb{R}_{+}\right)$is a solution to the integral equation

$u(x, y)=p+\int_{0}^{\alpha(x)} \int_{0}^{\beta(y)}\left[a(s, t) u(s, t)+\int_{0}^{s} \int_{0}^{t} b(s, t, \sigma, \eta) u(\sigma, \eta) d \sigma d \eta\right] d t d s, \quad x \geq 0, y \geq 0$.

If

$$
\lim _{x \rightarrow \infty}\left(\lim _{y \rightarrow \infty} \int_{0}^{\alpha(x)} \int_{0}^{\beta(y)}\left[a(s, t)+\int_{0}^{s} \int_{0}^{t} b(s, t, \sigma, \eta) d \sigma d \eta\right] d t d s\right)<\infty
$$

then $u$ is bounded.

Theorem 2.2. Assume that $p, a, b, \alpha, \beta$ are as in Theorem 2.1 and $g(r)$ is a positive continuous non-decreasing function for $r>0$ with $g(0)=0$ and $\int_{1}^{\infty} \frac{d t}{g(t)}=\infty$, if $u \in C\left(\mathbb{R}_{+} \times \mathbb{R}_{+}, \mathbb{R}_{+}\right)$satisfies for $x \geq 0, y \geq 0$

$u(x, y) \leq p(x, y)+\int_{0}^{\alpha(x)} \int_{0}^{\beta(y)}\left[a(s, t) g(u(s, t))+\int_{0}^{s} \int_{0}^{t} b(s, t, \sigma, \eta) g(u(\sigma, \eta)) d \sigma d \eta\right] d t d s$,

then

$$
u(x, y) \leq G^{-1}\left(G(p(x, y))+\int_{0}^{\alpha(x)} \int_{0}^{\beta(y)}\left[a(s, t)+\int_{0}^{s} \int_{0}^{t} b(s, t, \sigma, \eta) d \sigma d \eta\right] d t d s\right)
$$

where

$$
G(r)=\int_{1}^{r} \frac{d t}{g(t)}, \quad r \geq 0
$$

Proof. Assume $T_{1}, T_{2}>0$ is fixed and let

$$
z(x, y)=\int_{0}^{\alpha(x)} \int_{0}^{\beta(y)}\left[a(s, t) g(u(s, t))+\int_{0}^{s} \int_{0}^{t} b(s, t, \sigma, \eta) g(u(\sigma, \eta)) d \sigma d \eta\right] d t d s
$$

with the assumption on $a, b, \alpha, \beta$ imply that $z(x, y)$ is non-decreasing about $x$ and $y$. Hence for $x \in\left[0, T_{1}\right], y \in\left[0, T_{2}\right]$ we have 


$$
\begin{aligned}
z_{x y}(x, y) & =\alpha^{\prime}(x) \beta^{\prime}(y)[a(\alpha(x), \beta(y)) g(u(\alpha(x), \beta(y))) \\
& \left.+\int_{0}^{\alpha(x)} \int_{0}^{\beta(y)} b(\alpha(x), \beta(y), \sigma, \eta) g(u(\sigma, \eta)) d \sigma d \eta\right] \\
\leq & \alpha^{\prime}(x) \beta^{\prime}(y)[a(\alpha(x), \beta(y))(g(p(\alpha(x), \beta(y)))+g(z(\alpha(x), \beta(y)))) \\
& \left.+\int_{0}^{\alpha(x)} \int_{0}^{\beta(y)} b(\alpha(x), \beta(y), \sigma, \eta)(g(p(\sigma, \eta))+g(z(\sigma, \eta))) d \sigma d \eta\right] \\
& \leq g\left(p\left(T_{1}, T_{2}\right)+z\left(T_{1}, T_{2}\right)\right) \\
& \times\left(\alpha^{\prime}(x) \beta^{\prime}(y)\left[a(\alpha(x), \beta(y))+\int_{0}^{\alpha(x)} \int_{0}^{\beta(y)} b(\alpha(x), \beta(y), \sigma, \eta) d \sigma d \eta\right]\right) .
\end{aligned}
$$

Therefore, we write

$$
\frac{z_{x y}(x, y)}{g\left(p\left(T_{1}, T_{2}\right)+z\left(T_{1}, T_{2}\right)\right)} \leq \frac{\partial^{2}}{\partial x \partial y}\left(\int_{0}^{\alpha(x)} \int_{0}^{\beta(y)}\left[a(s, t)+\int_{0}^{s} \int_{0}^{t} b(s, t, \sigma, \eta) d \sigma d \eta\right] d t d s\right) .
$$

Noting that

$$
\frac{\partial}{\partial y}\left(\frac{z_{x}(x, y)}{g\left(p\left(T_{1}, T_{2}\right)+z\left(T_{1}, T_{2}\right)\right)}\right) \leq \frac{z_{x y}(x, y)}{g\left(p\left(T_{1}, T_{2}\right)+z\left(T_{1}, T_{2}\right)\right)} .
$$

We obtain

$$
\frac{\partial}{\partial y}\left(\frac{z_{x}(x, y)}{g\left(p\left(T_{1}, T_{2}\right)+z\left(T_{1}, T_{2}\right)\right)}\right) \leq \frac{\partial^{2}}{\partial x \partial y}\left(\int_{0}^{\alpha(x)} \int_{0}^{\beta(y)}\left[a(s, t)+\int_{0}^{s} \int_{0}^{t} b(s, t, \sigma, \eta) d \sigma d \eta\right] d t d s\right) .
$$

Integrating both sides of the above inequality with respect to $y$ from 0 to $y$ we get

$$
\frac{z_{x}(x, y)}{g\left(p\left(T_{1}, T_{2}\right)+z\left(T_{1}, T_{2}\right)\right)} \leq \frac{\partial}{\partial x}\left(\int_{0}^{\alpha(x)} \int_{0}^{\beta(y)}\left[a(s, t)+\int_{0}^{s} \int_{0}^{t} b(s, t, \sigma, \eta) d \sigma d \eta\right] d t d s\right),
$$

then integrating the above inequality with respect to $x$ from 0 to $x$ we have $(2.8)$

$$
G\left(p\left(T_{1}, T_{2}\right)+z\left(T_{1}, T_{2}\right)\right) \leq G\left(p\left(T_{1}, T_{2}\right)\right)+\int_{0}^{\alpha(x)} \int_{0}^{\beta(y)}\left[a(s, t)+\int_{0}^{s} \int_{0}^{t} b(s, t, \sigma, \eta) d \sigma d \eta\right] d t d s
$$


for $x \in\left[0, T_{1}\right], y \in\left[0, T_{2}\right]$.

In view of $\int_{1}^{\infty} \frac{d t}{g(t)}=\infty$, from (2.8), we have

$$
\left.p\left(T_{1}, T_{2}\right)+z\left(T_{1}, T_{2}\right)\right) \leq G^{-1}\left(G\left(p\left(T_{1}, T_{2}\right)\right)+\int_{0}^{\alpha(x)} \int_{0}^{\beta(y)}\left[a(s, t)+\int_{0}^{s} \int_{0}^{t} b(s, t, \sigma, \eta) d \sigma d \eta\right] d t d s\right) .
$$

Let $x=T_{1}, y=T_{2}$ in (2.9), we obtain

$\left.p\left(T_{1}, T_{2}\right)+z\left(T_{1}, T_{2}\right)\right) \leq G^{-1}\left(G\left(p\left(T_{1}, T_{2}\right)\right)+\int_{0}^{\alpha\left(T_{1}\right)} \int_{0}^{\beta\left(T_{2}\right)}\left[a(s, t)+\int_{0}^{s} \int_{0}^{t} b(s, t, \sigma, \eta) d \sigma d \eta\right] d t d s\right)$.

Due to $T_{1}, T_{2}$ are arbitrary and $u(x, y) \leq p(x, y)+z(x, y)$, we obtain $(2.7)$.

Corollary 2.3. Assume that $p, a, b, \alpha, \beta$ are as in Theorem 2.2. Suppose $u \in$ $C\left(\mathbb{R}_{+} \times \mathbb{R}_{+}, \mathbb{R}_{+}\right)$is a solution to the integral equation

$u(x, y)=p(x, y)+\int_{0}^{\alpha(x)} \int_{0}^{\beta(y)}\left[a(s, t) g(u(s, t))+\int_{0}^{s} \int_{0}^{t} b(s, t, \sigma, \eta) g(u(\sigma, \eta)) d \sigma d \eta\right] d t d s$,

for $x \geq 0, y \geq 0$. If $p$ is bounded and

$$
\lim _{x \rightarrow \infty}\left(\lim _{y \rightarrow \infty} \int_{0}^{\alpha(x)} \int_{0}^{\beta(y)}\left[a(s, t)+\int_{0}^{s} \int_{0}^{t} b(s, t, \sigma, \eta) d \sigma d \eta\right] d t d s\right)<\infty
$$

then $u$ is bounded.

\section{Basic Application}

In this section, we will present some basic applications of our results to obtain the bounds on the solution to the integral equation with time delay. We would like to develop a set of benchmark applications which may be used in the theory of partial differential and integral equations with time delay so we invite other researchers to contact us with their results for these cases, and perhaps forward us their own examples.

\subsection{Application:}

In order to exemplify the application of Theorem 2.1 we set up the bound on the solutions of partial integral equations of the form :

$$
u(x, y)=k(x, y)+\int_{0}^{\alpha(x)} \int_{0}^{\beta(y)}\left[G(x, y, s, t, u(s, t))+\int_{0}^{s} \int_{0}^{t} F(x, y, s, t, \sigma, \eta, u(\sigma, \eta)) d \sigma d \eta\right] d t d s
$$


where all the function are continuous on their respective domains of their definitions and

$$
\begin{gathered}
|k(x, y)| \leq p(x, y) \\
|G(s, t, u)| \leq a(s, t) u(s, t) \\
|F(s, t, \sigma, \eta, u(\sigma, \eta))| \leq b(s, t, \sigma, \eta) u(\sigma, \eta)
\end{gathered}
$$

for $x \geq 0, y \geq 0$ where $a, b, p, \alpha, \beta$ are as in Theorem 2.1 using the equations (3.2)(3.4) in the equation (3.1) then applying Theorem 2.1, we obtain the bound on the solution $u(x, y)$ to the equation (3.1).

In addition to this, in order to provide explicit bounds on the solution to partial differential equations of the form $u_{x y}=G(x, y, \alpha(s), \beta(y), u)$, one can use the integral inequalities which are obtain in Theorems 2.1 and 2.2.

\section{Concluding Remarks}

In concluding this paper, we have established some new generalized Pachpatte-type inequalities. As it can be seen from the present application, the results established are useful in researching both qualitative and quantitative properties for solutions to certain fractional order differential equations.

\section{R E F E R E N C E S}

1. D. Bainov and P. Simeonov: Integral Inequalities and Applications. Kluwer Academic Publishers, Dordrecht, 1992.

2. R. Bellman: The stability of solutions of linear differential equations. Duke Math. J., 10 (1943), 643-647.

3. I. BIHARI: generalization of a lemma of Bellman and its application to uniqueness problems of differential equations. Acta. Math. Acad. Sci. Hungar., 7 (1965), 81-94.

4. T. H. Gronwall: Note on the derivatives with respect to a parameter of the solutions of a system of differential equations. Ann. Math., 20 (1919), 292-296.

5. O. Lipovan: A retarded Gronwall-like inequality and its applications. J. Math. Anal. Appl., 252 (2000), 389-401.

6. B. G. Pachpatte: Inequalities for Differential and Integral Equations. Academic Press, New York, 1998.

7. B. G. Pachpatte: On some new inequalities related to certain inequalities in the theory of differential equations. J. Math. Anal. Appl. 189 (1995) 128-144.

8. B. G. PAChPATte: On some retarded integral inequalities and applications. J. Inequal. Pure Appl. Math., 3(2), Article 18, 2002. 
9. F. Usta and M. Z. SARIKAYA: Explicit bounds on certain integral inequalities via conformable fractional calculus. Cogent Mathematics, 4: 1277505, 2017.

10. F. Usta and M. Z. SARIKAyA: Some improvements of conformable fractional integral inequalities. International Journal of Analysis and Applications, 14(2), (2017), 162-166.

11. F. Usta and M. Z. SARIKAYA: On generalization of pachpatte type inequalities for conformable fractional integral. TWMS J. App. Eng. Math. 8(1), 2018, pp. 106-113.

12. F. Usta and M. Z. SARIKAYA: on generalization conformable fractional integral inequalities. FILOMAT. (In press).

Fuat Usta

Faculty of Science and Arts

Department of Mathematics

Düzce University, Düzce, Turkey

fuatusta@duzce.edu.tr

Mehmet Zeki Sarıkaya

Faculty of Science and Arts

Department of Mathematics

Düzce University, Düzce, Turkey

sarikayamz@gmail.com 